\title{
Influence of dynamic unbalance of wheelsets on the dynamic performance of high-speed cars
}

\author{
Yong $\mathrm{WANG}^{1 *}$, Hongjun $\mathrm{KANG}^{2}$, Xiaowen $\mathrm{SONG}^{3}$, Yaru LIANG ${ }^{1}$ \\ 1. Traction Power State Key Laboratory, Southwest Jiaotong University, Chengdu 610031, China \\ 2. Tangshan Railway Vehicle Co., Ltd., Tangshan 063030, China \\ 3. CSR Sifang Locomotive and Rolling Stock Co., Ltd., Qingdao 266111, China
}

\begin{abstract}
With further increasing in running speed of newer high-speed EMUs (electric multiple units), higher demand for wheelset dynamic balance is required. In order to study the influence of the dynamic unbalance of wheelset on the stability, ride quality, and curving performance for a high-speed car, a detailed dynamic model of a high-speed EMU car is established using the software SIMPACK. The analysis results indicate that the dynamic unbalance of the wheelset significantly influences the dynamic performance of the high-speed car. The increase in dynamic unbalance of the wheelset will decrease the hunting critical speed, worsen the vertical ride quality, and increase the wheelset lateral force, derailment coefficient, and wheel unloading ratio. Therefore, in order to improve the stability, ride quality, and running safety of high-speed car, the values of dynamic unbalance of wheelset should be strictly controlled in manufacturing, and periodically monitored in operation.
\end{abstract}

Key words: high-speed car; wheelset; dynamic unbalance; dynamic performance

(C) 2011 JMT. All rights reserved.

\section{Introduction}

$\mathrm{W}$ heelsets are the important components of the railway vehicle bogies, and the ride quality and running safety of the vehicles are influenced directly by the mass inequality of the wheelset. Because of the possible existence of microstructural non-uniformity in materials, wheel asymmetric local shapes, wheel profile inaccuracy caused by asymmetrical wear or wheel out-ofroundness, assembly errors of wheel and axle, and so on, the principal inertia axis through the gravity center of the wheelset may not coincide with the wheelset rotation axis. Therefore, the unbalanced centrifugal force will occur on the wheelset during rotating, due to the wheelset unbalance. The centrifugal force is one of the important influence factors causing bearing wear, vehicle noise and vibration, and even destructive accidents [1-4].

In recent years, along with the continuous development of railway transportation in China, raising the train operating speed becomes an important means to increase the competitiveness of the railway transportation. With the increase of train speed, the frequencies of periodic ex-

Received Jun. 12, 2011; revision accepted Aug. 8, 2011

*Corresponding author. E-mail: wyong@swjtu.edu.cn (Y. Wang) (C) 2011 JMT. All rights reserved

doi: 10.3969/j.issn.2095-087X.2011.03.001 citation forces will increase, which will seriously influence the stability, ride quality, and curving performance of vehicles, or even reduce the service life of the wheels, bearings, and other components of the vehicles. The unbalance of wheels is one of the periodic excitation sources, and the influence of the wheel balance on ride quality becomes very important because of the increasing speed of railway trains. In order to improve the running safety and ride comfort of vehicles, the dynamic unbalance values of the wheelsets should be strictly limited, especially for high speed trains.

Many countries in the world have paid great attention to the balance performance of wheelsets and the dynamic unbalance value of the wheelset has been controlled within a certain range according to some standards. For the wheelset of a passenger car, it is regulated in the UIC (International Union of Railways) standard and Japan Standard that the unbalance limit value of the wheelset should be decided by the maximum acceleration of the carbody or the bogie. The allowable unbalance value of the wheelset can be determined when the maximum acceleration of the carbody center reaches $1 \mathrm{~m} / \mathrm{s}^{2}$ or the bogie frame $10 \mathrm{~m} / \mathrm{s}^{2}$. According to the UIC standard, the maximum remaining unbalance value of the wheelset should be less than $50 \mathrm{gm}$ when the passenger car runs faster than $200 \mathrm{~km} / \mathrm{h}$ [5-6]. The Ministry of Railways of China has also promulgated the corresponding measures and technical standards to ensure the 
running performance of the speed-raising trains. According to the standard of TB/T2562-1995 [7], the remaining dynamic unbalance on the measuring face for each wheel after balance treatment should meet the allowable values listed in Table 1.

Table 1 The allowable dynamic unbalance

gm

\begin{tabular}{cc}
\hline $\begin{array}{c}\text { Running speed } \\
(\mathrm{km} / \mathrm{h})\end{array}$ & $\begin{array}{c}\text { Allowable dynamic unbalance } \\
\text { of each wheel }\end{array}$ \\
\hline$v \geq 200$ & $\leq 50$ \\
$200>v \geq 120$ & $\leq 75$ \\
\hline
\end{tabular}

Although we can determine the permissible value of the dynamic unbalance of the wheelset around the speed $200 \mathrm{~km} / \mathrm{h}$, referring to UIC standard and TB standard at present, it is difficult to determine the reasonable permissible value of the dynamic unbalance for the new type high-speed EMU because the highest running speed of the EMU has reached $350 \mathrm{~km} / \mathrm{h}$, and the corresponding rotational speed and the centrifugal force of the wheelset are further increased. Thus, the dynamic unbalance of the wheelset for the new type high-speed EMU should be given stricter limit. It is also necessary to study the influence of the dynamic unbalance of high-speed wheelset on dynamic performance by the numerical simulation and try to give suggestions for the limitation of wheelset dynamic unbalance values for the high-speed EMU.

Previous research is mostly focused on the dynamics of rotor-bearing system and the guarantee measures in design and manufacturing for the wheelset unbalance [815], and little attention has been paid to the effect of dynamic unbalance on vehicle dynamic performance. In this paper, taking a high-speed EMU car as an example, the influence of the dynamic unbalance of wheelset on the stability, ride quality, and curving performance of the car is studied, and the forced vibration responses and the frequency behavior caused by the dynamic unbalance are also investigated.

\section{Mathematical model}

Because of the existence of the dynamic unbalance, the principal inertia axis through the gravity center of a wheel does not coincide with the rotation axis; i.e., an eccentricity of the wheel exists. When the wheel rotates at a certain speed, the centrifugal force $(F)$ generated by the eccentricity of the wheel can be expressed as follows:

$$
F=M e(\pi n / 30)^{2},
$$

where $M$ is mass of wheel, in $\mathrm{kg}$; $e$ is eccentricity, namely the offset of the gravity center of wheel to the rotation axis, in $\mathrm{mm}$; and, $n$ is the rotation velocity of wheel, in $\mathrm{r} / \mathrm{min}$.
For a high-speed train running at speed $v$, the frequency of the periodic disturbing excitation caused by the dynamic unbalance of the wheel can be expressed as

$$
f=\frac{v}{7.2 \pi r},
$$

where $r$ is wheel rolling radius, in $\mathrm{m}$.

Eq. (1) indicates that the wheel rotating with a high speed will lead to a larger unbalance centrifugal force even if there is a small eccentricity, which will cause wheel and bearing wear, and vehicle system vibration.

A proper balancing mass $m_{\mathrm{b}}$ should be added to the wheel to eliminate the effect of the eccentricity and to balance the wheel. We presume that the distance from the mass $m_{\mathrm{b}}$ to the axis is $r_{\mathrm{b}}$ and the centrifugal force $F^{\prime}$ generated by $m_{\mathrm{b}}$ is equal in magnitude and opposite in direction to $F$ which is described as Eq. (1). Thus, there exists a relationship $M e=m_{\mathrm{b}} r_{\mathrm{b}}$. The product of unbalance mass $m_{\mathrm{b}}$ and rotation radius $r_{\mathrm{b}}$ can be used to express the dynamic unbalance of the wheel.

The wheelset is assembled by two wheels and one axle as a rotor revolving around the axis. Then the possible unbalance cases of the rigid rotational wheelset can be summarized as shown in Fig. 1.

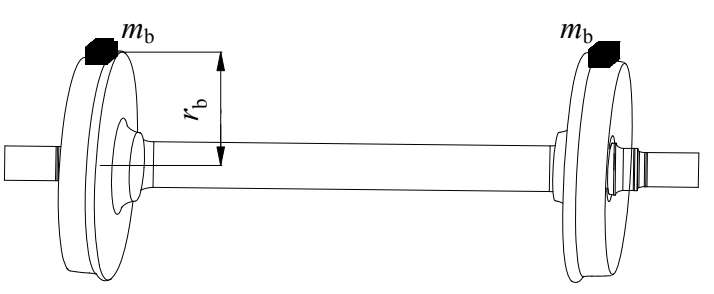

(a) Static unbalance

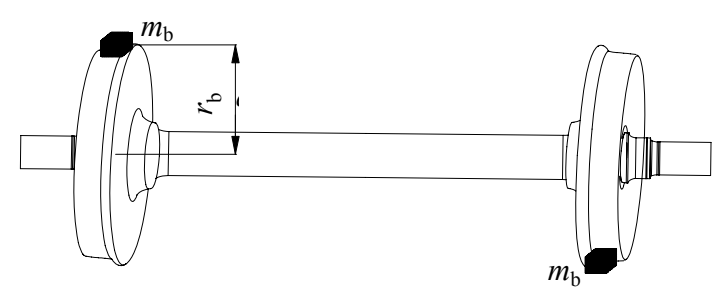

(b) Coupled unbalance

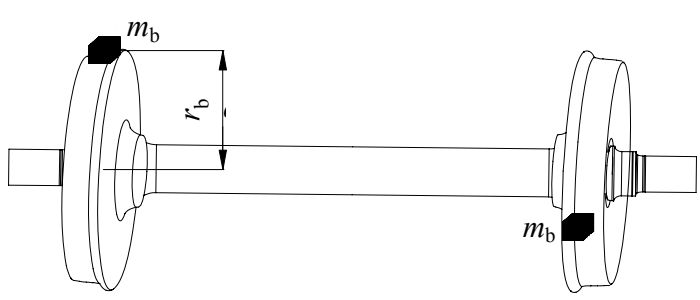

(c) Dynamic unbalance

Fig. 1 Possible unbalance states of wheelset 


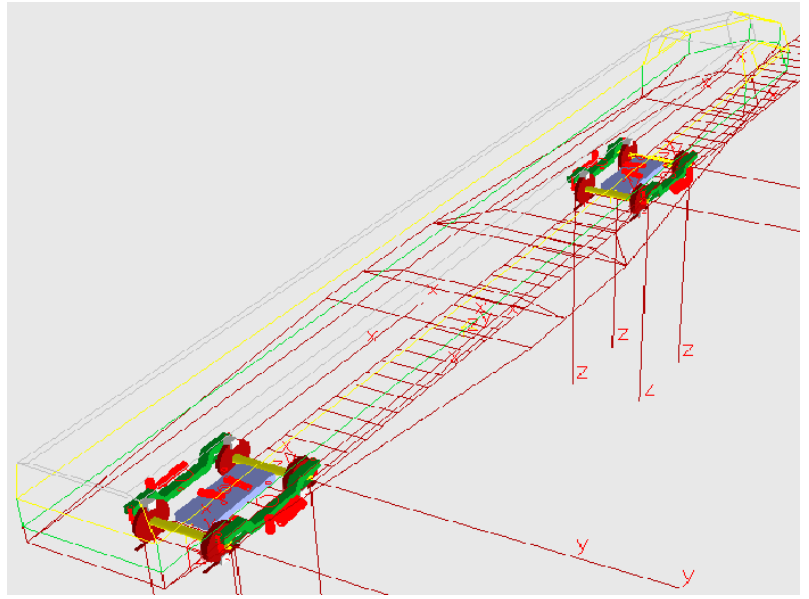

Fig. 2 Dynamic simulation model

As illustrated in Fig. 2, a detailed lateral and vertical coupled dynamic model of a high-speed car is established by using SIMPACK to study the influence of the above-mentioned dynamic unbalance of wheelset on the dynamic performance of the high-speed car. The simulation model is considered as a multiple rigid body system composed of a carbody, two bogie frames, two motors, four wheelsets, eight axle box guiding arms, and primary and secondary suspensions. The coordinate system of each body in the vehicle system is chosen to have its origin at the center of gravity of the body, and moves along the track center line at a constant forward speed $v$ in the $x$ direction. The $y$ axis is parallel to the track plane and directed to the right of the track and the $z$ axis is normal to the plane of the track and directed downward.

The track elasticity in the lateral and vertical directions is also considered in the mathematical model. In the dynamic calculations, the leading wheelset of the leading bogie in the traveling direction is defined as the first wheelset of the vehicle system. The total number of independent degrees of freedom of the vehicle system is 54 , which is listed in Table 2.

Table 2 Degrees of freedom of the model

\begin{tabular}{ccccccc}
\hline & Longitudinal & Lateral & Vertical & Roll & Pitch & Yaw \\
\hline Carbody & $X_{\mathrm{c}}$ & $Y_{\mathrm{c}}$ & $Z_{\mathrm{c}}$ & $\Phi_{\mathrm{c}}$ & $\theta_{\mathrm{c}}$ & $\psi_{\mathrm{c}}$ \\
Bogie frame & $X_{\mathrm{b} 1-2}$ & $Y_{\mathrm{b} 1-2}$ & $Z_{\mathrm{b} 1-2}$ & $\Phi_{\mathrm{b} 1-2}$ & $\theta_{\mathrm{b} 1-2}$ & $\psi_{\mathrm{b} 1-2}$ \\
Wheelset & $X_{\mathrm{w} 1-4}$ & $Y_{\mathrm{w} 1-4}$ & $\left(Z_{\mathrm{w} 1-4}\right)$ & $\left(\Phi_{\mathrm{w} 1-4}\right)$ & $\theta_{\mathrm{w} 1-4}$ & $\psi_{\mathrm{w} 1-4}$ \\
$\begin{array}{c}\text { Axle box } \\
\text { guiding arm }\end{array}$ & & & & $\theta_{\mathrm{a} 1-8}$ & \\
Motor & $X_{\mathrm{m} 1-2}$ & $Y_{m 1-2}$ & $Z_{\mathrm{m} 1-2}$ & $\Phi_{\mathrm{m} 1-2}$ & $\theta_{\mathrm{m} 1-2}$ & $\psi_{\mathrm{m} 1-2}$ \\
\hline
\end{tabular}

To consider the influence of the wheelset unbalance on dynamic performance, the equivalent masses denoting the wheelset unbalance values are added to the left and right wheels when establishing the dynamic model. The different wheelset unbalance can be constituted by different masses and different rotation radii. With the help of numerical calculation, the influence of the wheelset unbalance on the stability, ride quality and curving performance is studied. It is worth mentioning that the effects of the three unbalance states shown in Fig. 1 on the dynamic performance have similar trends. The results given below show the greatest effect.

\section{Numerical simulation}

\subsection{Hunting stability}

The calculation result of the influence of the coupled unbalance of the wheelset on the critical speed is shown in Fig. 3. It can be seen that with the increase of dynamic unbalance of wheelset, the critical speed of the

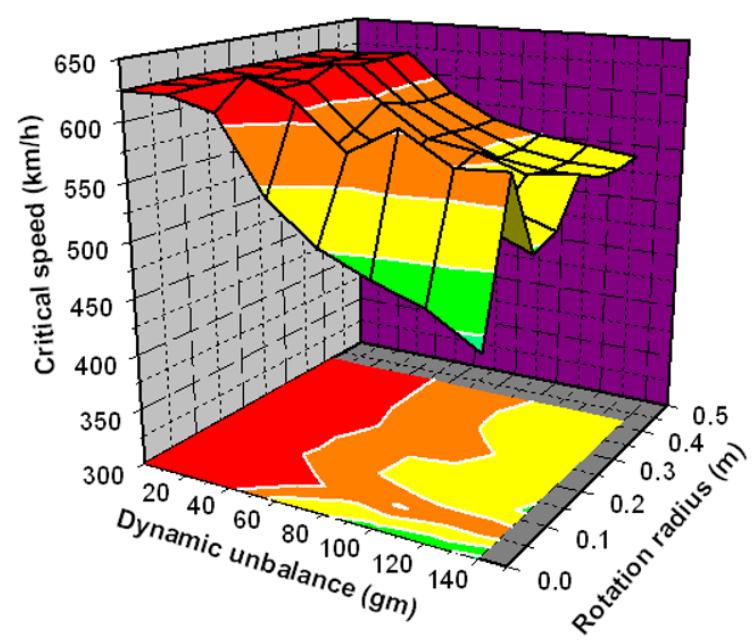

Fig. 3 Influence of dynamic unbalance of wheelset on the critical speed 
vehicle system reduces obviously when the dynamic unbalance of wheelset is more than $45 \mathrm{gm}$. In addition, the rotation radius of the unbalance mass has little influence on the critical speed of the vehicle system, except in the case that the rotation radius of the unbalance mass is too small, which means that a larger mass is added to the wheelset at the position close to the axis in the dynamic model, and the vehicle system has the lowest critical speed. The main reason is that, at a certain speed, the disturbance force caused by the dynamic unbalance of wheelset mainly depends on the product of the unbalance mass and the rotation radius as expressed in formula (1).

\subsection{Ride quality}

The results about the influence of the static unbalance of the wheelset on the lateral and vertical ride indexes are shown in Fig. 4 and Fig. 5, respectively.

The results illustrate that, with the increase of dynamic unbalance of wheelset, the lateral ride index of carbody increases slightly. It is apparent that the rotation radius of the unbalance mass has little influence on lateral ride index when the rotation radius is relatively large (e.g., in the range of $0.2-0.45 \mathrm{~m}$ ). The lateral ride index obviously increases when the rotation radius of the unbalance mass approaches zero.

The dynamic unbalance of wheelset has a greater effect on the vertical ride index of the carbody. We can see that the vertical ride index increases as the dynamic unbalance of wheelset increases. This is caused by the additional disturbance force produced by the dynamic unbalance mass when the wheelset rotates. Also, there is

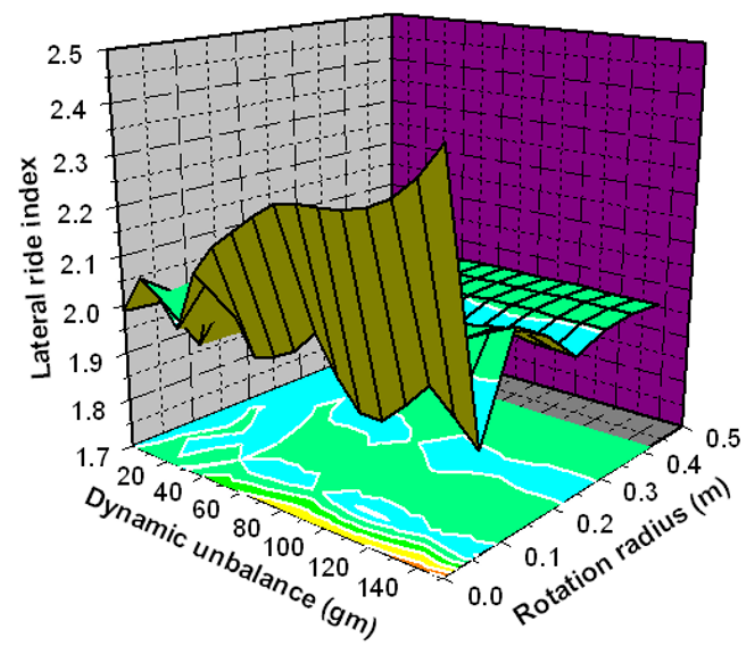

Fig. 4 Influence of dynamic unbalance of wheelset on lateral ride index

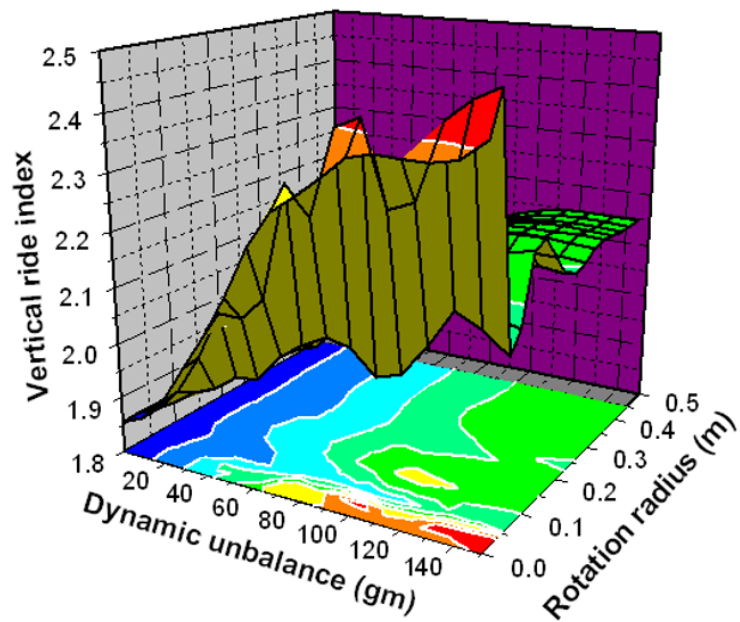

Fig. 5 Influence of dynamic unbalance of wheelset on vertical ride index

the same trend that the rotation radius of the unbalance mass has little influence on vertical ride index when the rotation radius is in the range of large values. The system has the largest vertical ride index when the rotation radius of the unbalance mass approaches zero, and the vertical ride index increases evidently with the dynamic unbalance increasing.

\subsection{Curving performance}

Fig. 6 to Fig. 11 illustrate the effects of coupled unbalance of wheelset on lateral force of wheelset, wheel unloading rate, vertical wheel/rail force, and derailment ratio of the first wheelset. The car operating condition is that the curve radius is $7000 \mathrm{~m}$, super-elevation is $133 \mathrm{~mm}$ and the running speed is $350 \mathrm{~km} / \mathrm{h}$. It can be seen from the results that, the curving performance of the car becomes worse, and the derailment ratio, wheel unloading rate and vertical wheel/rail force increase with the increasing of the wheelset dynamic unbalance. The rotation radius of the unbalance mass has little influence on curving performance when the rotation radius is in the range of large values, for the same reason that the disturbance force caused by the dynamic unbalance of the wheelset mainly depends on the product of the unbalance mass and the rotation radius.

In fact, the worst condition, such as the rotation radius close to zero and the unbalance mass too large usually does not occur, because there are strict error control standards for the mass of the wheelset in manufacturing and operation, even though the mass may change due to wheel wear and flatting. 


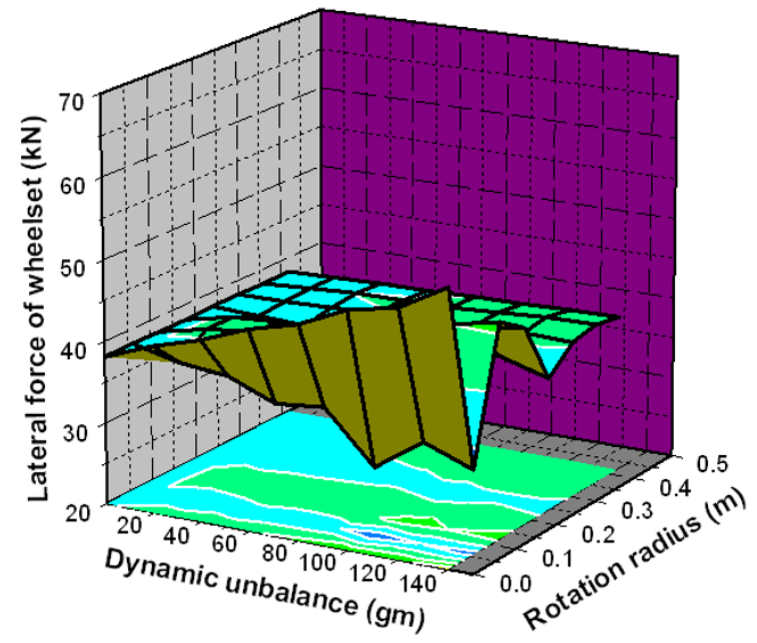

Fig. 6 Influence of dynamic unbalance of wheelset on lateral wheelset force

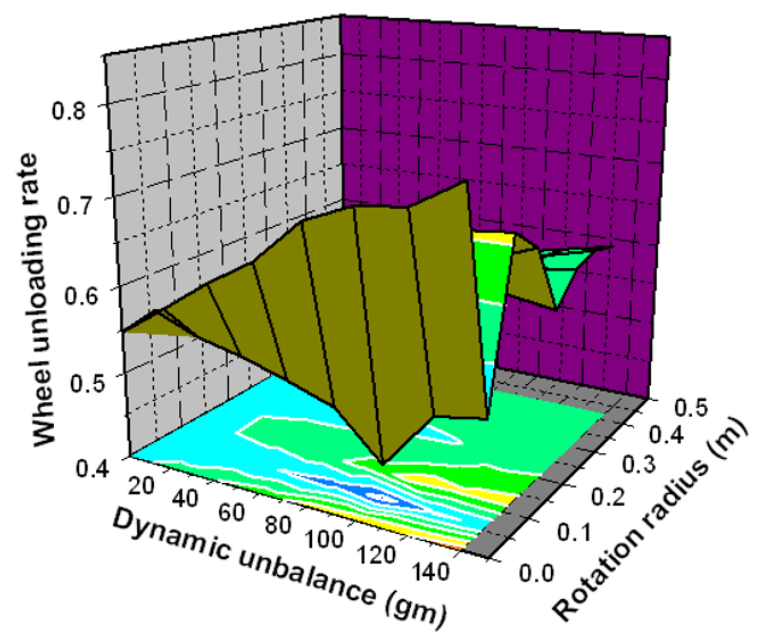

Fig. 7 Influence of dynamic unbalance of the wheelset set on wheel unloading rate

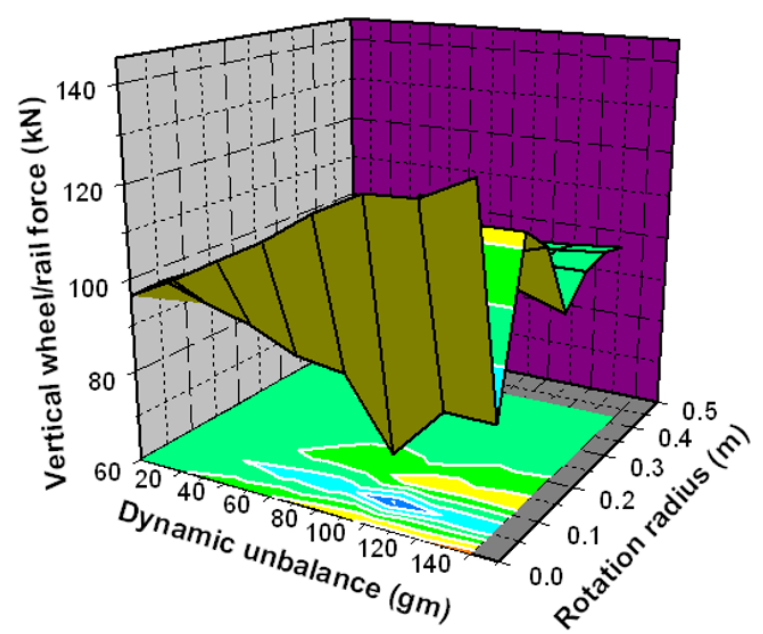

Fig. 8 Influence of dynamic unbalance of the wheelset on left vertical wheel/rail force

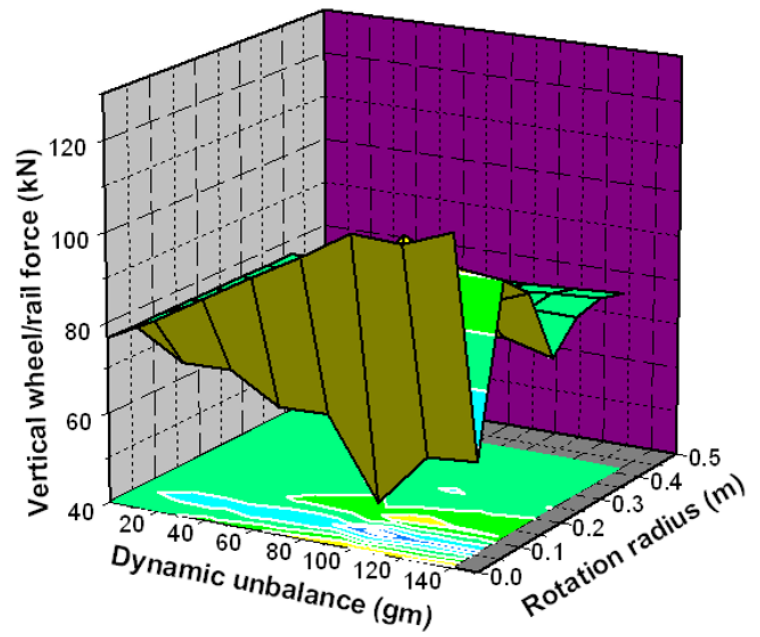

Fig. 9 Influence of dynamic unbalance of the wheelset on right vertical wheel/rail force

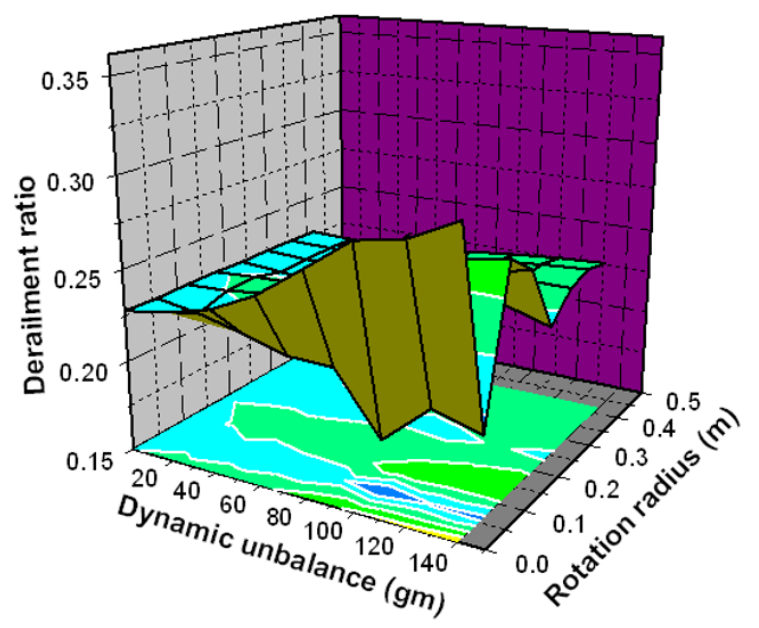

Fig. 10 Influence of dynamic unbalance of the wheelset on left wheel derailment ratio

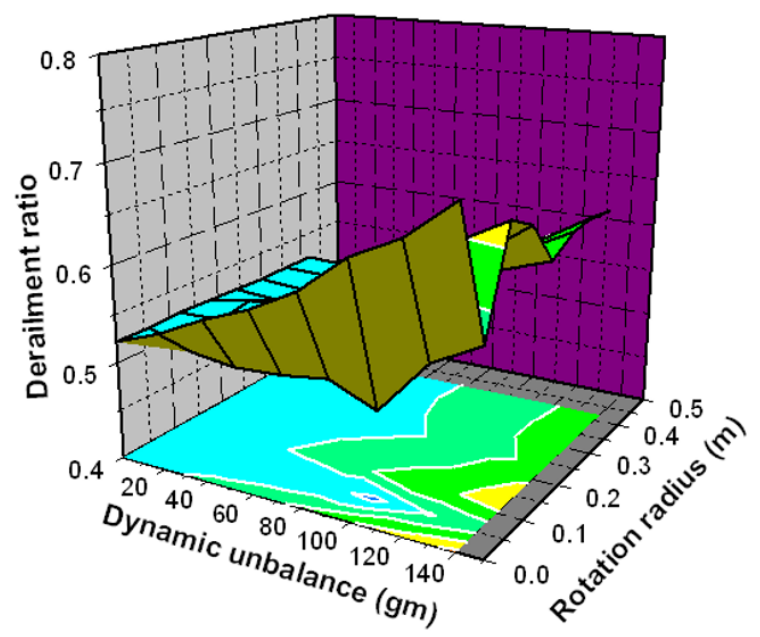

Fig. 11 Influence of dynamic unbalance of the wheelset on right wheel derailment ratio 


\subsection{Frequency analysis}

According to Eq. (2), the frequencies of the forced vibration caused by the dynamic unbalance of wheelset under different running speeds are obtained as shown in Table 3, where the nominal rolling radius is $0.46 \mathrm{~m}$.

At the same time, the natural frequencies of the vehicle system rigid body modes of the vehicle system and the first torsional frequency of the bogie frame, the first lateral and vertical bending frequencies, are calculated and shown in Fig. 12 and Fig. 13, respectively.

Table 3 Forced vibration frequency caused by the dynamic unbalance of wheelset

\begin{tabular}{ccccccc}
\hline $\begin{array}{c}\text { Speed } \\
(\mathrm{km} / \mathrm{h})\end{array}$ & 100 & 200 & 300 & 400 & 500 & 600 \\
\hline $\begin{array}{c}\text { Excitation } \\
\text { frequency }(\mathrm{Hz})\end{array}$ & 9.61 & 19.22 & 28.84 & 38.45 & 48.06 & 57.67 \\
\hline
\end{tabular}

It can be seen from Fig. 12 that, if the running speed is around $300-400 \mathrm{~km} / \mathrm{h}$ for the high-speed car, the natural frequencies of lateral vibrations of the vehicle system are lower than the bogie hunting frequency, and no resonance occurs. It is seen from Fig. 13 that the natural frequencies of vertical vibrations of the vehicle system are less than the excitation frequency caused by the wheelset dynamic unbalance in the running speed range of $300-400 \mathrm{~km} / \mathrm{h}$, and no resonance occurs. However, at the speed of $355 \mathrm{~km} / \mathrm{h}$, the vibration frequency caused by dynamic unbalance is close to the first torsional frequency of the bogie frame and bogie torsional resonance appears. This may cause bogie frame fatigue problems and should be avoided through the strict control of the dynamic unbalance of the wheelset.

According to the vibration frequency analysis, it is necessary to strictly limit the dynamic unbalance of the wheelsets in order to avoid the occurring of resonance of the vehicle system.
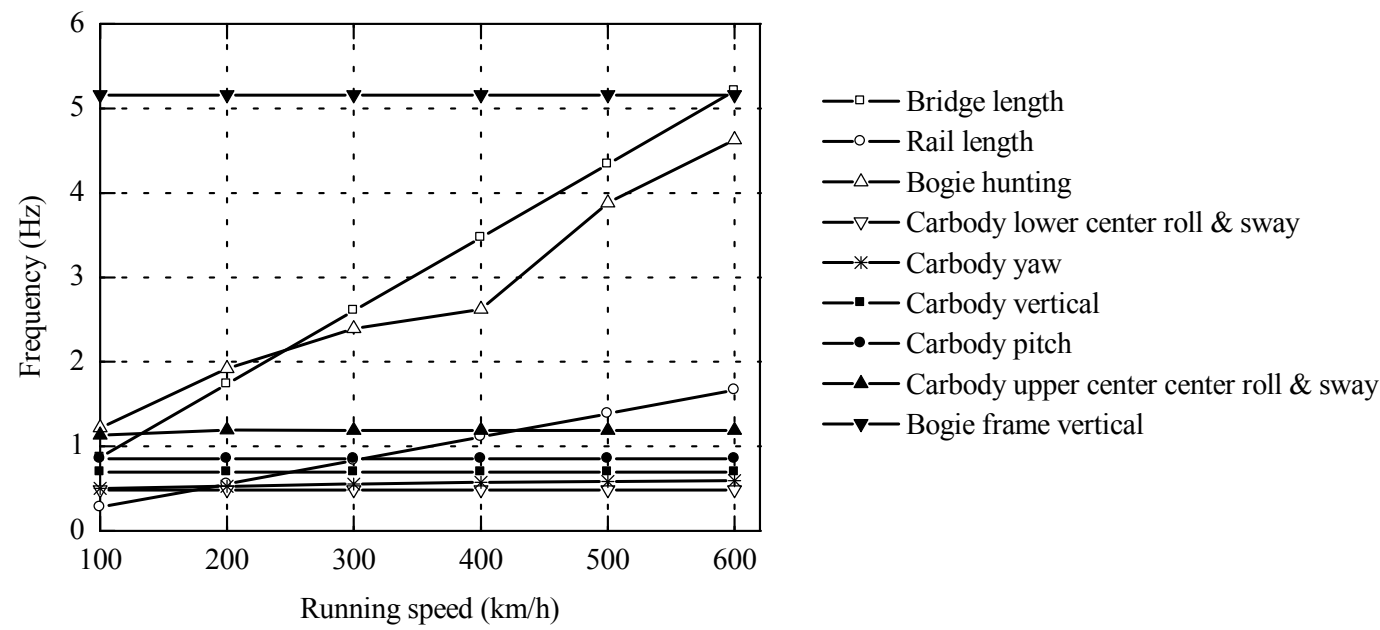

Fig. 12 Vibration spectrum of low frequency components (less than $6 \mathrm{~Hz}$ )

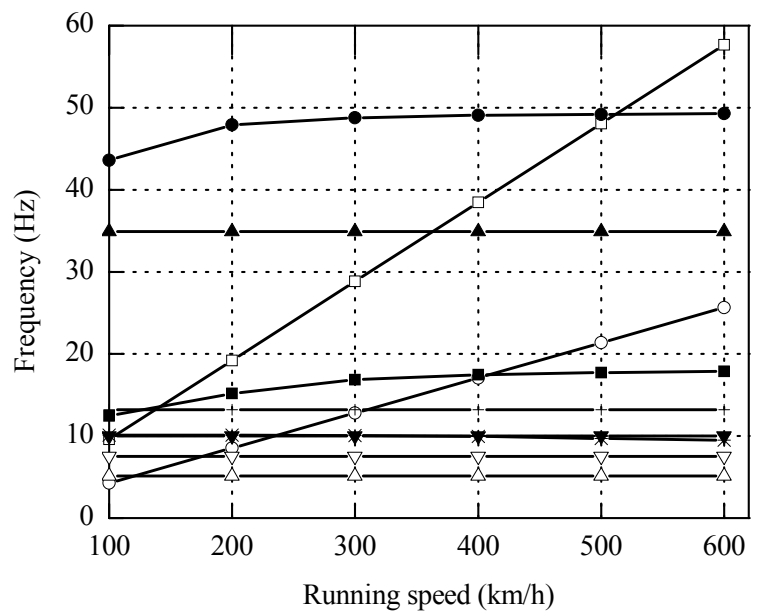

- - Dynamic unbalance of wheelset

- - Track slab length

$-\triangle-$ Bogie frame verical

$-\nabla-$ Bogie frame pitch

- $*$ - Bogie frame roll

- - Bogie frame lateral

$-\bullet-$ Bogie frame yaw

- $\mathbf{\Delta - B o g i e}$ frame 1 st order torsional

$\mathbf{- \nabla}$-Carbody 1 st order lateral bending

-+- Carbody 1 st order vertical bending

Fig. 13 Vibration spectrum of high frequency components (higher than $5 \mathrm{~Hz}$ ) 


\section{Conclusions}

The influences of the dynamic unbalance of wheelsets on dynamic performance including stability, ride quality, and curving performance of high-speed car are studied. The possible resonance vibrations of the vehicle system caused by the wheelset dynamic unbalance are also analyzed. The following conclusions can be drawn:

(1) The increase of dynamic unbalance of the wheelset will decrease the critical speed. The critical speed decreases when the dynamic unbalance of the wheelset is more than $45 \mathrm{gm}$. At the same time, the vertical vibration performance is also getting worse. Thus, it is suggested that the dynamic unbalance of the wheelset should be limited less than $25 \mathrm{gm}$.

(2) Dynamic unbalance of the wheelset has large effect on the vertical ride index of the carbody. As the dynamic unbalance increases, the vertical ride index increases, while the lateral ride index increases slightly.

(3) The curving behavior of the car deteriorates with the increase in the wheelset dynamic unbalance, and the vertical wheel/rail force, derailment ratio and wheel unloading rate of the leading wheelset increase.

(4) At the speed about $355 \mathrm{~km} / \mathrm{h}$, the vibration frequency caused by dynamic unbalance is close to the first torsional frequency of the bogie frame and bogie torsional resonance appears, which may cause bogie frame fatigue problems.

In general, a periodic disturbing excitation is exerted on the wheelset because of the existence of the dynamic unbalance. The inertia vibration caused by dynamic unbalance of the wheelset becomes stronger with the increasing of dynamic unbalance and running speed. The force between the wheel and rail and the vertical ride index are also increased. Thus, the service life of wheel and bearing may be shortened after a long time running. Therefore, with the increase in the running speed, the dynamic unbalance of wheelset should be strictly limited in manufacturing and regularly inspected during operation to ensure the stability, running safety, and ride comfort for a high speed car.

\section{Acknowledgements}

This work was supported by the National High Technology Research and Development Program of China (2009AA110303-06), the National S\&T Program in the 11th Five-Year Plan Period (2009BAG12A02-B02-2), the Program for New Century Excellent Talents in Universities (NCET-10-0664), and the Key Program of the Ministry of Railways (2010J003-E).

\section{References}

[1] S. Kaewunruen, A.M. Remennikov, Effect of a large asymmetrical wheel burden on flexural response and failure of railway concrete sleepers in track systems, Engineering Failure Analysis, 2008, 15(8): 1065-1075.

[2] M. Ishida, T. Ban, K. Iida, et al., Effect of moderating friction of wheel/rail interface on vehicle/track dynamic behaviour, Wear, 2008, 265(9-10): 1497-1503.

[3] D.V. Nguyen, K.D. Kim, P. Warnitchai, Simulation procedure for vehicle-substructure dynamic interactions and wheel movements using linearized wheel-rail interfaces, Finite Elements in Analysis and Design, 2009, 45(5): 341-356.

[4] S. Zakharov, I. Goryacheva, V. Bogdanov, et al., Problems with wheel and rail profiles selection and optimization, Wear, 2008, 265(9-10): 1266-1272.

[5] B.M. Wang, H.J. Zhang, The dynamic balance of wheelsets for high speed cars, Diesel Locomotive, 1995(9): 2427 (in Chinese).

[6] S.J. Tao, The dynamic balance of wheels for railway cars, Rolling Stock, 1993, 31(5): 15-18 (in Chinese).

[7] TB/T2562-1995, Testing methods for the static balance of wheel and dynamic balance of wheelset (in Chinese).

[8] Y. Chen, X.Y. Liu, The dynamic balance technology of wheelsets for high speed passenger cars and the improvement, Rolling Stock, 2000, 38(11): 11-13 (in Chinese).

[9] L.J. Du, L.N. Zhang, The new dynamic balance technology of wheelsets for high speed cars, Science \& Technology Information, 2008(30): 419-421 (in Chinese).

[10] L.P. Yang, The dynamic balance of wheelsets for high speed cars, Rolling Stock, 1995, 33(9): 37-40 (in Chinese).

[11] W.L. Wang, Dynamic balance of multiple unit driving wheelset, Diesel Locomotive, 2009(7): 15-17 (in Chinese).

[12] F.W. Kang, The New Technique of the Dynamic Balance of the Wheelset of the Rolling Stock [Dissertation], Chengdu: Southwest Jiaotong University, 2004.

[13] S.P. Harsha, Nonlinear dynamic response of a balanced rotor supported by rolling element bearings due to radial internal clearance effect, Mechanism and Machine Theory, 2005, 26(1): 47-66.

[14] S.P. Harsha, Nonlinear dynamic analysis of a high-speed rotor supported by rolling element bearings, Journal of Sound and Vibration, 2006, 290(1-2): 65-100.

[15] S.P. Harsha, K. Sandeep, R. Prakash, The effect of speed of balanced rotor on nonlinear vibrations associated with ball bearings, International Journal of Mechanical Sciences, 2003, 45(4): 725-740.

(Editor: Junsi LAN) 